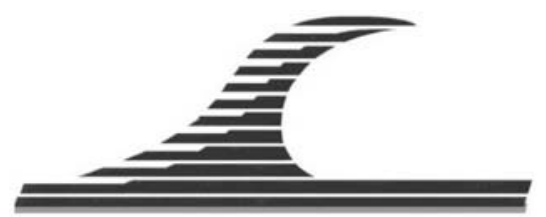

Revue Paralia, Volume 11 (2018) pp n01.1-n01.14

Mots-clés : Vagues non-linéaires, Hydrodynamique côtière,

Simulation des vagues, Modélisation numérique, Modèles de

vagues, Fonctions de base radiales.

(C) Editions Paralia CFL

\title{
Développement d'un modèle numérique non-linéaire et dispersif pour la propagation des vagues en zone côtière
}

\author{
Cécile RAOULT $^{1,2}$, Michel BENOIT ${ }^{3}$, Marissa L. YATES ${ }^{1,4}$
}

1. Laboratoire d'Hydraulique Saint-Venant, Université Paris-Est, Unité de recherche commune EDF R\&D, Cerema, Ecole de Ponts ParisTech, 6 quai Watier, BP 49, 78401 Chatou cedex, France.

2. EDF R\&D, Laboratoire National d'Hydraulique et Environnement - LNHE, 6 quai Watier, BP 49, F-78401 Chatou cedex, France.

3. Aix-Marseille Université, Institut de Recherche sur les Phénomènes Hors-Equilibre (IRPHE), UMR 7342- CNRS, Aix-Marseille Université, Ecole Centrale Marseille, 49 rue Frédéric Joliot-Curie, BP 146, F-13384 Marseille Cedex 13, France.

4. Cerema, Division technique Eau, mer et fleuves, 134 rue de Beauvais, 60280 Margny-les-Compiègne, France.

cecile.raoult@edf.fr ; benoit@irphe.univ-mrs.fr ; marissa.yates-michelin@cerema.fr

\section{Résumé :}

Les effets non-linéaires et dispersifs étant particulièrement importants pour les vagues en zone côtière, nous étudions et développons un modèle potentiel complètement nonlinéaire et dispersif résolvant les équations d'Euler-Zakharov qui régissent l'évolution temporelle de la position et du potentiel des vitesses à la surface libre.

La formulation mathématique ainsi que sa mise en œuvre numérique sont exposées, avec la présentation de la méthode d'extension du domaine d'une à deux dimensions d'espace horizontales. Les capacités non-linéaires et dispersives de la version 1DH du modèle sont démontrées à travers l'application à deux cas tests : d'abord, la génération et la propagation des harmoniques libres et liées associées aux vagues régulières créées par un générateur de vagues de type piston sur un fond plat d'après les expériences de CHAPALAIN et al. (1992), puis la propagation de vagues irrégulières au-dessus d'une barre sous-marine d'après les expériences de BECQ-GIRARD et al. (1999). La bonne représentation des transferts d'énergie entre les différentes composantes harmoniques montre la capacité et la précision du modèle à représenter les effets dispersifs et nonlinéaires d'ordres élevés.

Article issu d'une sélection des XIVèmes Journées Nationales Génie Côtier Génie Civil qui se sont tenues à Toulon du 29 juin au ler juillet 2016. Soumis le 9 janvier 2017, accepté le 16 mai 2018, en ligne le 18 juin 2018.

La seule version examinée est celle écrite en français. La ou les autres versions n'étant pas examinées par le comité de rédaction de la revue, sont donc publiées sous l'entière responsabilité du ou des auteurs.

A TRANSLATED VERSION IN ENGLISH IS AVAILABLE ONLINE

Pour citer cet article :

RAOULT C., BENOIT M., YATES M.L. (2018). Développement d'un modèle numérique non-linéaire et dispersif pour la propagation des vagues en zone côtière. Revue Paralia, Vol. 11, pp n01.1-n01.14.

DOI: https://doi.org/10.5150/revue-paralia.2018.n01 
Le développement d'une version 2DH du modèle a été testé pour simuler la propagation de vagues régulières sur une marche immergée semi-circulaire agissant comme une lentille convergente, afin de reproduire deux des expériences de WHALIN (1971). Les premiers résultats obtenus utilisant des fonctions de base radiales pour calculer les dérivées dans le plan horizontal montrent la capacité du modèle de simuler des cas de bathymétries variables en $2 \mathrm{DH}$. Cette méthode semble prometteuse en vue de l'application à des cas réalistes.

\section{Introduction}

La transformation des vagues depuis le large jusqu'à la plage fait intervenir un grand nombre de processus physiques, incluant la réfraction et le levage (shoaling), des effets dissipatifs dus à la friction sur le fond ou au déferlement, la diffraction et la réflexion sur des structures, îles ou hauts-fonds, etc. La modélisation précise de l'ensemble de ces processus est loin d'être triviale, compte tenu notamment du fait que la profondeur d'eau relative pour les vagues $\mu=k h$ (où $k$ est le nombre d'onde et $h$ la profondeur d'eau) varie très significativement entre le large et la plage. Par ailleurs, les effets nonlinéaires, quantifiés à l'aide du paramètre de cambrure $\varepsilon=k H / 2$ ou de hauteur de vague relative $H / h$ (où $H$ est la hauteur caractéristique des vagues), peuvent devenir importants en zone côtière avec la diminution de la profondeur d'eau.

Pour traiter correctement ces deux aspects, les modèles numériques de simulation des vagues doivent posséder des capacités dispersives et non-linéaires élevées. Certaines approches de modélisation utilisées couramment en ingénierie côtière et portuaire, fondées par exemple sur l'équation de pente douce (mild slope equation) de BERKHOFF (1972) dans sa version originale ou ses extensions, font le choix de négliger les effets non-linéaires pour la propagation des vagues. Les modèles de type Boussinesq, Serre ou Green-Naghdi permettent une prise en compte partielle des effets non-linéaires et/ou dispersifs (e.g. MADSEN et al., 1991 ; NWOGU, 1993 ; KIRBY, 2003 ; CHAZEL et al., 2011). Cependant, ces modèles ne sont que partiellement dispersifs et/ou partiellement non-linéaires, même dans le cas de développements d'ordres élevés (MADSEN et al., 2002, 2006 ; BENOIT \& CHAZEL, 2013).

Dans le but de disposer d'un outil de simulation précis, les auteurs ont choisi de développer un modèle complètement dispersif et non-linéaire, fondé sur les équations surfaciques d'Euler-Zakharov (ZAKHAROV, 1968) dans un cadre potentiel (YATES \& BENOIT, 2015). BENOIT et al. (2014) et RAOULT et al. $(2014,2016)$ ont présenté plusieurs cas de validation de la version 1DH $(x, z)$ dans des conditions de vagues nondéferlantes. Ici, les avancées récentes sur le développement et la validation de ce modèle numérique sont exposées et discutées.

Nous rappelons brièvement les fondements mathématiques du modèle dans la partie 2 , puis donnons dans la partie 3 un aperçu des méthodes numériques utilisées. Dans la partie 4, deux cas de validation 1DH mettant en jeu des effets non-linéaires importants 
sont présentés et analysés en détail. Une première application sur un cas 2DH (i.e. 3D) est présentée en partie 5 avec la simulation des expériences de WHALIN (1971) pour deux conditions de vagues incidentes sur une bathymétrie provoquant une convergence des vagues. Les conclusions et perspectives sont résumées dans la partie 6.

\section{Présentation du modèle mathématique}

En considérant l'écoulement irrotationnel d'un fluide homogène, non visqueux et de masse volumique constante, une modélisation mathématique potentielle est adoptée. Le système est décrit par le potentiel des vitesses $\Phi(\vec{x}, z, t)$, qui vérifie l'équation de Laplace dans tout le domaine fluide (équation de conservation de la masse), avec la notation $\vec{x}=(x, y)$. Cette équation est complétée par deux conditions aux limites (non-linéaires), dynamique et cinématique, à la surface libre en $z=\eta(\vec{x}, t)$, une condition d'imperméabilité au fond en $z=-h(\vec{x}, t)$ et des conditions aux frontières latérales de type Dirichlet ou Neumann. Dans les applications présentées ici, le fond est constant en temps, mais le modèle peut aussi être utilisé pour étudier des vagues de type tsunami générées par des mouvements du fond marin (voir BENOIT et al. 2014).

En supposant la continuité de la colonne d'eau entre le fond et la surface, on peut définir le potentiel des vitesses à la surface libre $\Psi(\vec{x}, t)=\Phi(\vec{x}, z=\eta(\vec{x}, t), t)$ et les conditions aux limites à la surface libre deviennent (ZAKHAROV, 1968) :

$$
\begin{aligned}
& \frac{\partial \eta}{\partial t}=-\nabla \eta \cdot \nabla \Psi+\widetilde{w}\left(1+(\nabla \eta)^{2}\right) \\
& \frac{\partial \Psi}{\partial t}=-g \eta-\frac{1}{2}(\nabla \Psi)^{2}+\frac{1}{2} \widetilde{w}^{2}\left(1+(\nabla \eta)^{2}\right)
\end{aligned}
$$

où $\widetilde{w}(\vec{x}, t) \equiv \frac{\partial \Phi}{\partial z}(\vec{x}, z=\eta, t)$ est la composante verticale de la vitesse à la surface libre.

Le système (1-2) ne fait intervenir que des variables surfaciques. Cependant, l'intégration en temps de ces équations nécessite de connaître $\widetilde{w}$, qui doit être estimée à partir des deux quantités $\eta$ et $\psi$, problème appelé "Dirichlet-to-Neumann" (DtN).

\section{Présentation de la résolution numérique}

La simulation numérique du système (1-2) nécessite : un schéma d'intégration en temps, une méthode de résolution du problème DtN et une méthode de calcul des dérivées premières et secondes en espace. Les détails des méthodes numériques pour la version 1DH $(x, z)$ du modèle sont disponibles dans YATES \& BENOIT (2015) et RAOULT et al. $(2014,2016)$; seuls les points principaux sont rappelés ici. Pour l'avancement en temps, un schéma de Runge-Kutta explicite d'ordre 4, avec un pas de temps constant, est utilisé.

Le problème DtN est résolu en utilisant une méthode spectrale sur la verticale (TIAN \& SATO, 2008) pour trouver la solution du problème aux limites de Laplace pour le potentiel $\Phi$ dans tout le domaine. Un changement de coordonnée verticale défini par : 
$s(\vec{x}, z, t)=\frac{2 z+h(\vec{x}, t)-\eta(\vec{x}, t)}{h(\vec{x}, t)+\eta(\vec{x}, t)}$

permet de se ramener à un domaine de hauteur constante, avec $s=-1$ au fond et $s=+1$ à la surface libre. Ensuite, une approximation spectrale du potentiel de vitesse est appliquée, en utilisant une base de polynômes de Tchebychev de première espèce $T_{n}(s)$ sur $[-1,+1]$ :

$\Phi(\vec{x}, z, t)=\varphi(\vec{x}, s(\vec{x}, z, t), t) \approx \sum_{n=0}^{N_{T}} a_{n}(\vec{x}, t) T_{n}(s)$

La décomposition est tronquée à un ordre $N_{T}$, valant 5 à 10 comme valeur optimale pour les applications pratiques, comme montré par BENOIT et al. (2014) et RAOULT et al. (2014, 2016). Dans la version 1DH du modèle, des schémas aux différences finies d'ordre 4 sont utilisés pour calculer les dérivées spatiales, avec un pas d'espace variable dans le cas général. En pratique, la résolution du problème aux valeurs limites de Laplace à un instant donné se ramène finalement à la résolution d'un système linéaire portant sur les coefficients $a_{n}(\vec{x})$ pour $n=0,1, \ldots, N_{T}$. La vitesse verticale en surface libre s'obtient alors par :

$\widetilde{w}(\vec{x}, t)=\frac{\partial \Phi}{\partial z}(\vec{x}, z=\eta, t)=\frac{\partial s}{\partial z} \frac{\partial \varphi}{\partial s}(\vec{x}, s=1, t) \approx \frac{2}{h(\vec{x}, t)+\eta(\vec{x}, t)} \sum_{n=1}^{N_{T}} n^{2} a_{n}(\vec{x}, t)$

Afin d'aborder des cas plus réalistes, avec des configurations bathymétriques complexes et des vagues d'incidences multiples, le modèle a été étendu à deux dimensions horizontales (i.e. en 3D) en utilisant une méthode de fonctions de base radiales (RBF) (WRIGHT \& FORNBERG, 2006) sur une discrétisation par nuage de points dans le plan $(x, y)$ afin de s'adapter facilement à tout type de géométrie, tout en gardant une représentation spectrale sur la verticale. La dérivée d'une fonction $f$ est approximée en un nœud $\overrightarrow{x_{l}}$ comme une combinaison linéaire des valeurs de la fonction $f$ au $n-1$ plus proches voisins ( $n$ étant le nombre de nœuds dans la cellule ou "stencil") :

$L(f)\left(\overrightarrow{x_{l}}\right)=\sum_{k=1}^{n} w_{l, k}^{L} f\left(\overrightarrow{x_{k}}\right)$

avec $L$ un opérateur différentiel linéaire quelconque et les $w_{l, k}^{L}$ les inconnues à déterminer pour chaque opérateur $L$ et chaque nœud $\overrightarrow{x_{l}}$. Pour cela on utilise un ensemble de fonctions tests formant une base, pour lesquelles on impose que l'approximation soit exacte. Ici on choisit des fonctions radiales, centrées aux nœuds du stencil $\overrightarrow{x_{k}, k=1, n}$ : $\varphi_{k}(\vec{x})=\Phi\left(\left\|\vec{x}-\overrightarrow{x_{k}}\right\|\right)$. Cela conduit à résoudre un système linéaire de $n$ équations à $n$ inconnues dont la formulation matricielle est la suivante :

$\left[\begin{array}{cccc}\Phi\left(\left\|\overrightarrow{x_{1}}-\overrightarrow{x_{1}}\right\|\right) & \Phi\left(\left\|\overrightarrow{x_{2}}-\overrightarrow{x_{1}}\right\|\right) & \ldots & \Phi\left(\left\|\overrightarrow{x_{n}}-\overrightarrow{x_{1}}\right\|\right) \\ \Phi\left(\left\|\overrightarrow{x_{1}}-\overrightarrow{x_{2}}\right\|\right) & \Phi\left(\left\|\overrightarrow{x_{2}}-\overrightarrow{x_{2}}\right\|\right) & & \vdots \\ \vdots & & \ddots & \vdots \\ \Phi\left(\left\|\overrightarrow{x_{1}}-\overrightarrow{x_{n}}\right\|\right) & \cdots & \cdots & \Phi\left(\left\|\overrightarrow{x_{n}}-\overrightarrow{x_{n}}\right\|\right)\end{array}\right]\left[\begin{array}{c}w_{l, 1}^{L} \\ w_{l, 2}^{L} \\ \vdots \\ w_{l, n}^{L}\end{array}\right]=\left[\begin{array}{c}L \Phi\left(\left\|\vec{x}-\overrightarrow{x_{1}}\right\|\right)\left(\overrightarrow{x_{\imath}}\right) \\ L \Phi\left(\left\|\overrightarrow{x^{2}}-\overrightarrow{x_{2}}\right\|\right)\left(\overrightarrow{x_{\imath}}\right) \\ \vdots \\ L \Phi\left(\left\|\vec{x}-\overrightarrow{x_{n}}\right\|\right)\left(\overrightarrow{x_{\imath}}\right)\end{array}\right]$ 
Avec des nœuds fixes, ce système est résolu, une seule fois en début de simulation, pour chacun des nœuds du domaine et pour chaque opérateur différentiel. Par la suite, la fonction radiale utilisée est la fonction multiquadrique (WRIGHT \& FORNBERG, 2006) :

$\Phi\left(\left\|\vec{x}-\overrightarrow{x_{k}}\right\|\right)=\sqrt{\left\|\vec{x}-\overline{x_{k}}\right\|^{2}+c^{2}}$

où $c$ est le paramètre de forme. Plus la valeur de $c$ est élevée, plus la multiquadrique est aplatie, ce qui a pour effet de diminuer l'erreur d'approximation, mais en contrepartie dégrade le conditionnement de la matrice et donc augmente la difficulté de résolution du système linéaire et les erreurs d'arrondi.

\section{Validations de la version $1 \mathrm{DH}$ du modèle numérique}

\subsection{Dynamique d'un train de vagues généré par un batteur piston sur fond plat}

CHAPALAIN et al. (1992) ont étudié la propagation de vagues générées par le mouvement sinusoïdal d'un batteur piston sur un fond plat. Ce cas test consiste à simuler l'essai A correspondant à un piston se déplaçant d'une amplitude maximale de $e=7,8 \mathrm{~cm}$, à une période de $T=2,5 \mathrm{~s}$, dans une profondeur d'eau de $h=0,4 \mathrm{~m}$. D'après la relation de dispersion linéaire des vagues, on peut estimer la longueur d'onde des vagues générées à $L=4,74 \mathrm{~m}$. Pour la simulation numérique, on impose à la frontière gauche du modèle (côté batteur), une vitesse homogène sur la verticale, avec une variation sinusoïdale en temps. La frontière droite est suffisamment loin du batteur pour négliger les réflexions. On laisse les vagues se propager pendant 16 périodes (soit $40 \mathrm{~s}$ ) avec un pas de temps $\Delta t=T / 40=0,0625 \mathrm{~s}$. L'ordre maximal des polynômes de Tchebychev est fixé à $N_{T}=7$ et on utilise un maillage régulier en $x$, avec $\Delta x=0,1 \mathrm{~m}$.

Une fois le régime stationnaire atteint, une analyse harmonique du signal temporel d'élévation de surface libre évalue les amplitudes et les phases des différentes harmoniques (Figure 1a). Le modèle représente correctement le transfert d'énergie entre les différentes harmoniques, avec les bonnes longueurs de battement, due la présence simultanée de composantes libres et liées des harmoniques à 2,3 et 4 fois la fréquence de mouvement du batteur. L'écart observé pour la deuxième harmonique (2f) après $x=19 \mathrm{~m}$, dont l'amplitude diminue légèrement dans les expériences, est probablement dû aux effets dissipatifs qui ne sont pas pris en compte par le modèle. L'évolution spatiale de la différence de phase entre la première et la seconde harmonique (Figure 1b), oscillant entre $-\pi / 2$ et $+\pi / 2$ avec la même longueur de battement que les amplitudes, est également bien reproduite par le modèle. Les deux premières harmoniques sont en phase quand l'amplitude de la première harmonique est maximale et celle de la seconde minimale, et vice versa. 


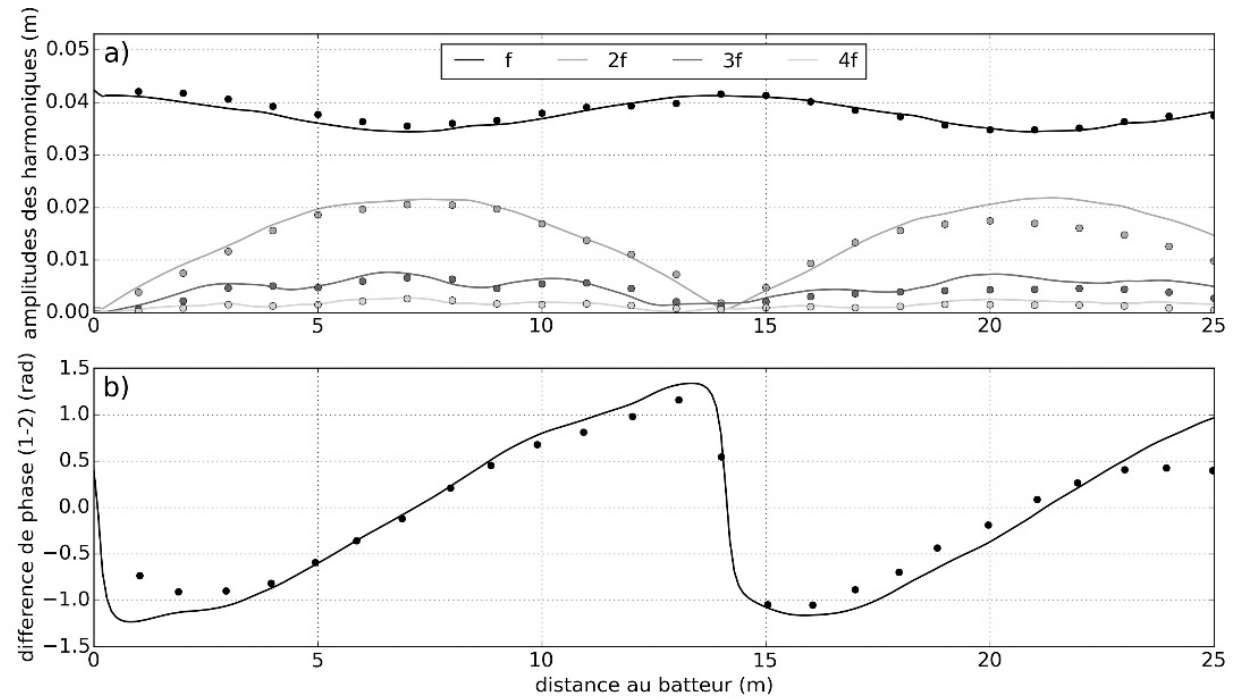

Figure 1. Evolution spatiale a) des amplitudes des quatre premières harmoniques d'élévation de surface libre pour le cas A de CHAPALAIN et al. (1992) et b) de la

différence de phase entre la première et la seconde harmonique. Les résultats expérimentaux sont représentés par les points et les résultats des simulations par les lignes continues.

\subsection{Propagation de vagues irrégulières sur une barre sous-marine}

Ce cas test a pour but de simuler la propagation de vagues irrégulières au-dessus d'une barre sous-marine d'après les expériences de BECQ-GIRARD et al. (1999). Le profil bathymétrique (Figure 2) a été spécialement conçu afin d'étudier les effets non-linéaires en eau peu profonde. Expérimentalement, une série de vagues irrégulières est générée à l'aide d'un piston selon un spectre JONSWAP avec un facteur d'élancement $\gamma=3,3$, de période pic $T_{p}=2,39 \mathrm{~s}$ et de hauteur significative $H_{m 0}=3,4 \mathrm{~cm}$ par $0,65 \mathrm{~m}$ de fond au large du profil bathymétrique (essai 26 de BECQ-GIRARD et al. (1999), sans déferlement sur la barre sous-marine). L'élévation de surface libre est mesurée par 16 sondes pendant $40 \mathrm{~min}$ avec un pas d'échantillonnage de $\Delta t=0,07 \mathrm{~s}$.

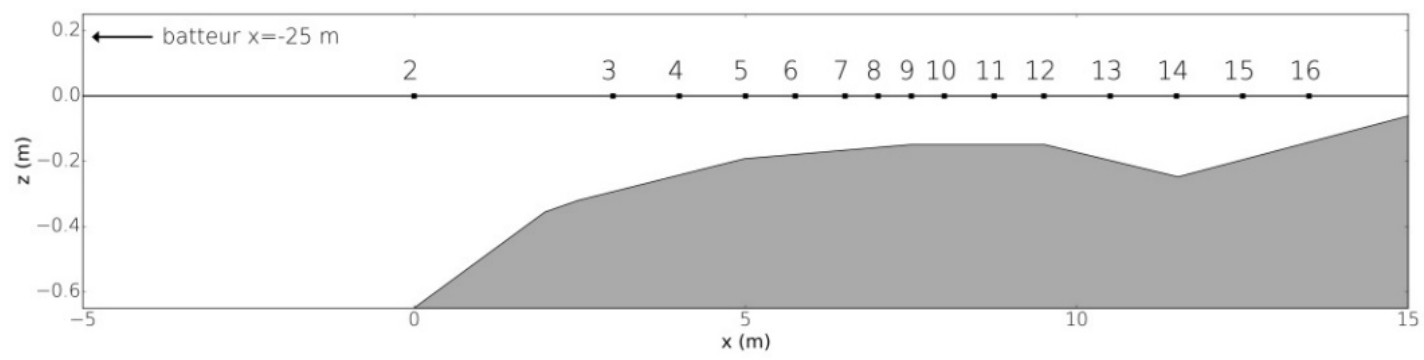

Figure 2. Bathymétrie et positions des sondes de mesure de vagues pour les expériences de BECQ-GIRARD et al. (1999). 
Dans la simulation, le domaine de calcul s'étend de $x=-5 \mathrm{~m}$ à $+25 \mathrm{~m}$, le début de la barre se situant à $x=0 \mathrm{~m}$. Le domaine est discrétisé de manière régulière avec un pas d'espace $\Delta x=0,05 \mathrm{~m}$, et $N_{T}=7$. Pour forcer le modèle sur la limite côté batteur, on utilise la série temporelle de surface libre mesurée à la sonde 2. Le potentiel associé est reconstruit à partir d'une analyse spectrale de cette surface libre en utilisant la théorie linéaire des vagues, et imposé sur la frontière gauche du domaine. Une zone de relaxation de $5 \mathrm{~m}$ de large est ajoutée pour la génération dans laquelle une correction de la position de la surface libre et du potentiel des vitesses est appliquée. De l'autre côté du domaine, une zone de relaxation de $10 \mathrm{~m}$ de large est également ajoutée pour absorber les vagues. La durée simulée est de 2380 s, soit environ 39,7 min avec un pas de temps identique à celui d'échantillonnage des sondes $\Delta t=0,07 \mathrm{~s}$.

Les spectres de variance de la surface libre montrent un bon accord avec les mesures (e.g. Figure 3, sondes 2, 9, 15 et 16). On observe en particulier un transfert d'énergie qui s'effectue du pic principal (à la fréquence $f_{p}$ ) vers les hautes fréquences avec l'apparition de pics dans le spectre de la sonde 9 aux fréquences harmoniques $\left(2 f_{p}, 3 f_{p}\right.$, etc.) et une bonne représentation des amplitudes des pics, même pour la cinquième harmonique. Juste après la barre, le transfert d'énergie s'inverse, avec un transfert principalement dirigé vers la seconde harmonique. On remarque bien, dans le spectre de la sonde 15, la quasi-disparition des pics aux harmoniques 3, 4 et 5 ainsi que l'augmentation du pic de l'harmonique 2. Finalement, dans le spectre de la sonde 16, le pic de la troisième harmonique est de nouveau bien marqué du fait de la diminution de la profondeur d'eau sur la partie terminale du profil bathymétrique. L'évolution de la partie basses fréquences du spectre est également bien reproduite par le modèle numérique.
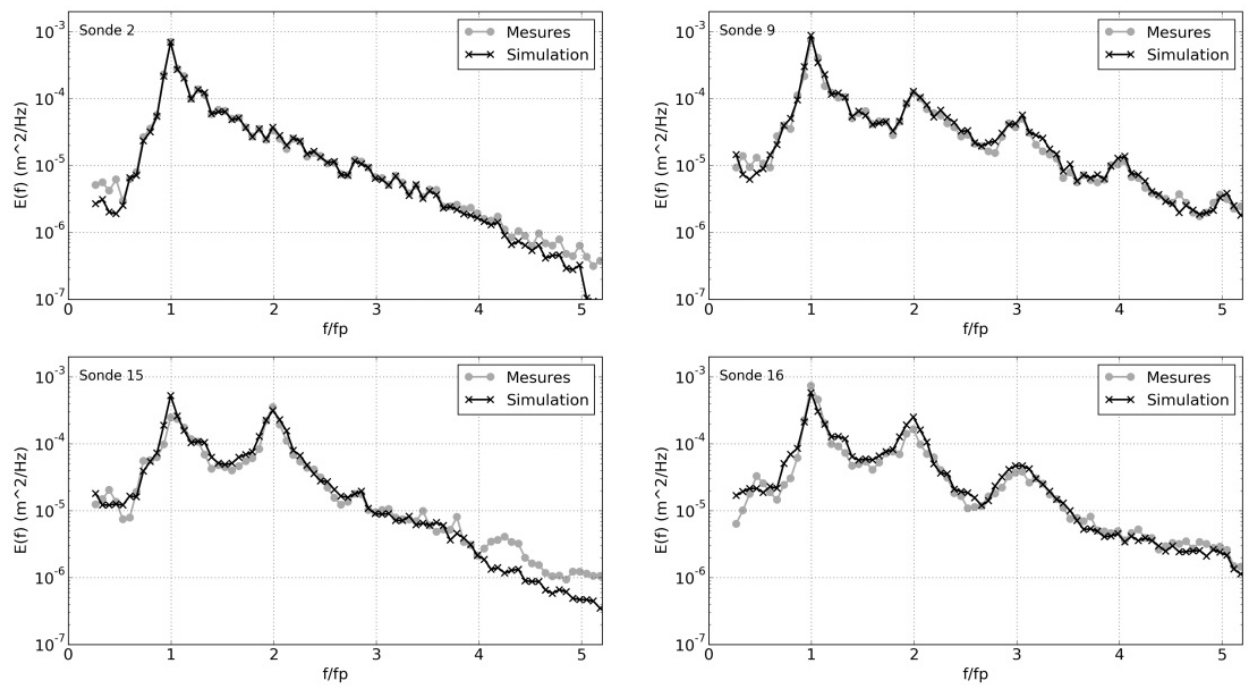

Figure 3. Comparaison des spectres de variance de la surface libre mesurés (en gris) et simulés (en noir) à quatre sondes (2, 9, 15 et 16) pour l'essai 26 de BECQ-GIRARD et al. (1999). 

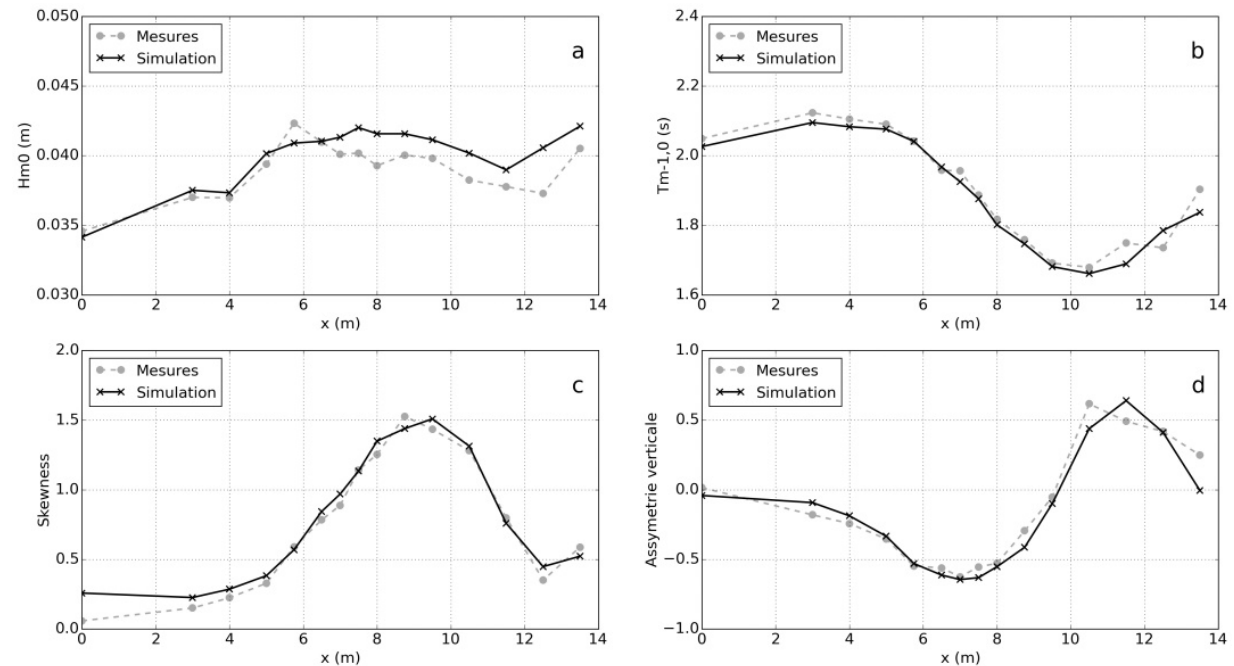

Figure 4. Comparaison des paramètres d'état de mer mesurés (en gris) et simulés (en noir) le long du profil bathymétrique des expériences de BECQ-GIRARD et al. (1999) :

a) hauteur significative des vagues, b) période moyenne des vagues, c) skewness (asymétrie horizontale) et d) asymétrie verticale.

Le modèle représente bien l'évolution le long du profil bathymétrique des paramètres intégraux de l'état de mer, comme la hauteur significative $H_{m 0}$ (Figure 4a) et la période énergétique moyenne $T_{m-1,0}$ (Figure $4 \mathrm{~b}$ ) qui sont calculées à partir des moments du spectre de variance. Les effets des non-linéarités se traduisent par une augmentation de la hauteur significative avec la diminution de la profondeur d'eau due au shoaling et une diminution dans le creux du profil bathymétrique alors que la période moyenne commence par décroître quand l'énergie est transférée vers les plus hautes fréquences puis augmente lorsque le transfert s'inverse et persiste. D'autre part, deux paramètres permettant de caractériser l'asymétrie des vagues ont été analysés : la skewness (Figure 4c) et l'asymétrie verticale (Figure 4d), définies respectivement par :

$\lambda_{3}=\frac{\left\langle(\eta-\langle\eta\rangle)^{3}\right\rangle}{m_{o}^{3 / 2}}=\frac{\sum_{m=-\infty}^{+\infty} \sum_{n=-\infty}^{+\infty} \operatorname{Re}\left[B_{m, n}\right]}{m_{o}^{3 / 2}} \quad A=\frac{\sum_{m=-\infty}^{+\infty} \sum_{n=-\infty}^{+\infty} \operatorname{Im}\left[B_{m, n}\right]}{m_{o}^{3 / 2}}$

où $B_{m, n}=B\left(f_{m}, f_{n}\right)$ est le bispectre, défini comme la transformée de Fourier de la fonction d'auto-corrélation d'ordre 3 de l'élévation de surface libre. Ces deux paramètres rendent compte respectivement de l'asymétrie crête/creux et front/face arrière des vagues. Sur la partie plate avant le profil bathymétrique, où les vagues sont faiblement non-linéaires, ces paramètres ont des valeurs proches de zéro, mais évoluent ensuite avec le profil bathymétrique en bon accord avec les mesures, traduisant un développement de l'asymétrie des profils de vagues à la fois par rapport à un plan horizontal et par rapport à un plan vertical. 


\section{Application et validation de la version 2DH du modèle numérique}

Ce cas test, basé sur les expériences de WHALIN (1971), met en œuvre une bathymétrie en forme de marche semi-circulaire qui joue le rôle d'une lentille convergente pour les vagues (Figure 5.a). Expérimentalement, des vagues régulières sont générées par un piston dans un bassin de 25,63 m de long et 6,096 $\mathrm{m}$ de large, dans une profondeur de $0,4572 \mathrm{~m}$. Le profil bathymétrique utilisé dans la simulation est le même que celui utilisé par SHAO \& FALTINSEN (2014). Deux conditions de vagues sont simulées, le premier cas correspond à des vagues régulières de période $T=2 \mathrm{~s}$ et d'amplitude $a=0,0075 \mathrm{~m}$. Dans le second cas la période reste identique, mais l'amplitude est augmentée $a=0,0106 \mathrm{~m}$. Le potentiel des vitesses correspondant est imposé à la frontière gauche du modèle. Le domaine de calcul comprend la zone d'intérêt de $0 \mathrm{~m}$ à $25 \mathrm{~m}$ à laquelle on a ajouté une zone de relaxation de 3,91 m de long (soit une longueur d'onde) afin d'absorber les vagues réfléchies sur la marche et d'en diminuer la réflexion au niveau de la frontière gauche. Une zone de relaxation de 7,5 m de long (soit approximativement trois longueurs d'onde) a été ajoutée du côté de la frontière droite afin d'absorber les vagues. Les frontières latérales sont considérées comme des parois imperméables. Le domaine a été discrétisé par un nuage de points non-structuré de 60716 nœuds (approximativement espacés de $0,06 \mathrm{~m}$ ). On simule une durée de $36 \mathrm{~s}$ (soit 18 périodes) avec un pas de temps constant $\Delta t=T / 75=0,0267 \mathrm{~s}$. Le nombre de nœuds par stencil est de $n=13$ (i.e. le stencil de chaque nœud est composé de ce nœud et de ses 12 plus proches voisins) et la constante de forme prise à $c=1$, après quelques tests de sensibilité des résultats aux valeurs de ces deux paramètres.

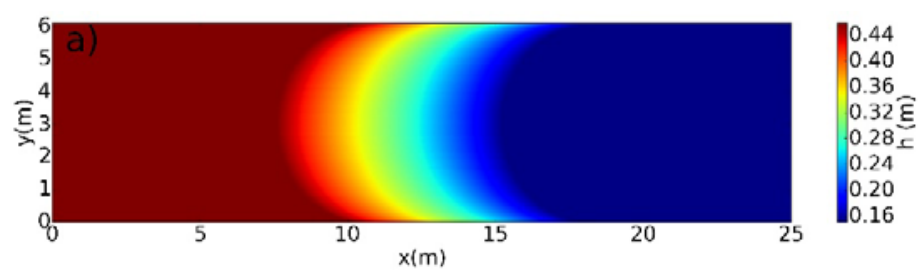

b)

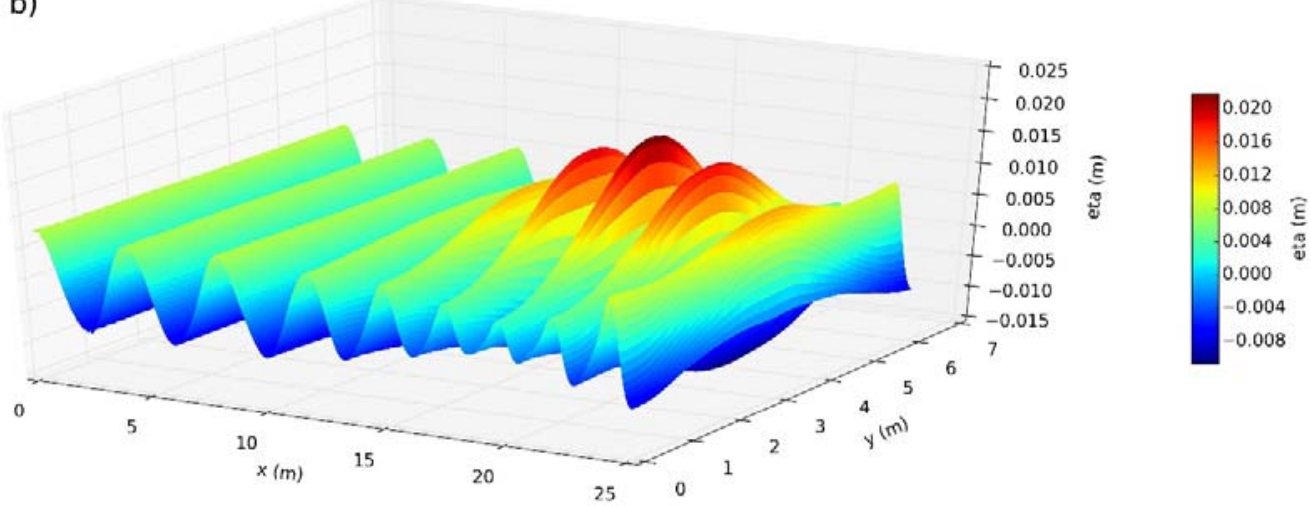

Figure 5. Bathymétrie (a) et position de la surface libre (b) simulée à l'instant final $(t \approx 36,045$ s) pour l'expérience de WHALIN (1971) avec a $=0.0075 \mathrm{~m}$. 
Le profil de la surface libre à l'instant final dans le premier cas (Figure 5b) montre que, dans la partie de profondeur constante avant la marche, les vagues sont quasiment invariantes en $y$ alors qu'une fois passée la marche la structure du champ de vagues est tridimensionnelle avec des effets non-linéaires importants. L'augmentation de l'amplitude de la surface libre dans la partie peu profonde est une conséquence de l'effet combiné du shoaling, de la diffraction et de la réfraction induits par le profil bathymétrique. Une analyse harmonique des séries temporelles de surface libre suivant l'axe central du bassin permet d'obtenir l'évolution spatiale des amplitudes des trois premières harmoniques pour le premier cas simulé (Figure 6). La simulation donne des résultats en bon accord avec les résultats expérimentaux. L'amplitude de l'harmonique deux est légèrement sous-estimée dans la partie la plus profonde du domaine $(x<10 \mathrm{~m})$, probablement du fait de la méthode linéaire de génération des vagues dans le modèle. Néanmoins, dans la zone de convergence $(x \approx 20 \mathrm{~m})$ les variations des amplitudes des harmoniques sont bien reproduites, notamment la forte augmentation des amplitudes des harmoniques deux $(2 f)$ et trois $(3 f)$ du fait du transfert d'énergie provenant de la première harmonique. Même avec ces transferts d'énergie vers les deuxième et troisième harmoniques, l'amplitude de la première harmonique reste constante (avant la zone d'absorption) après la marche. Cet effet intéressant est expliqué par la compensation de la perte d'énergie pour la première harmonique par la concentration de l'énergie liée à la réfraction autour de la bosse dont l'amplitude atteint ainsi une sorte de plateau. Pour le second cas simulé, lorsque l'amplitude de la vague incidente est augmentée (Figure 7), les effets non-linéaires sont plus importants. L'amplitude de l'harmonique deux atteint environ deux tiers de l'amplitude de la première harmonique dans la zone de convergence alors qu'elle n'atteignait que la moitié dans le premier cas. 
Développement d'un modèle numérique non-linéaire et dispersif pour la propagation des vagues en zone côtière : n01.11

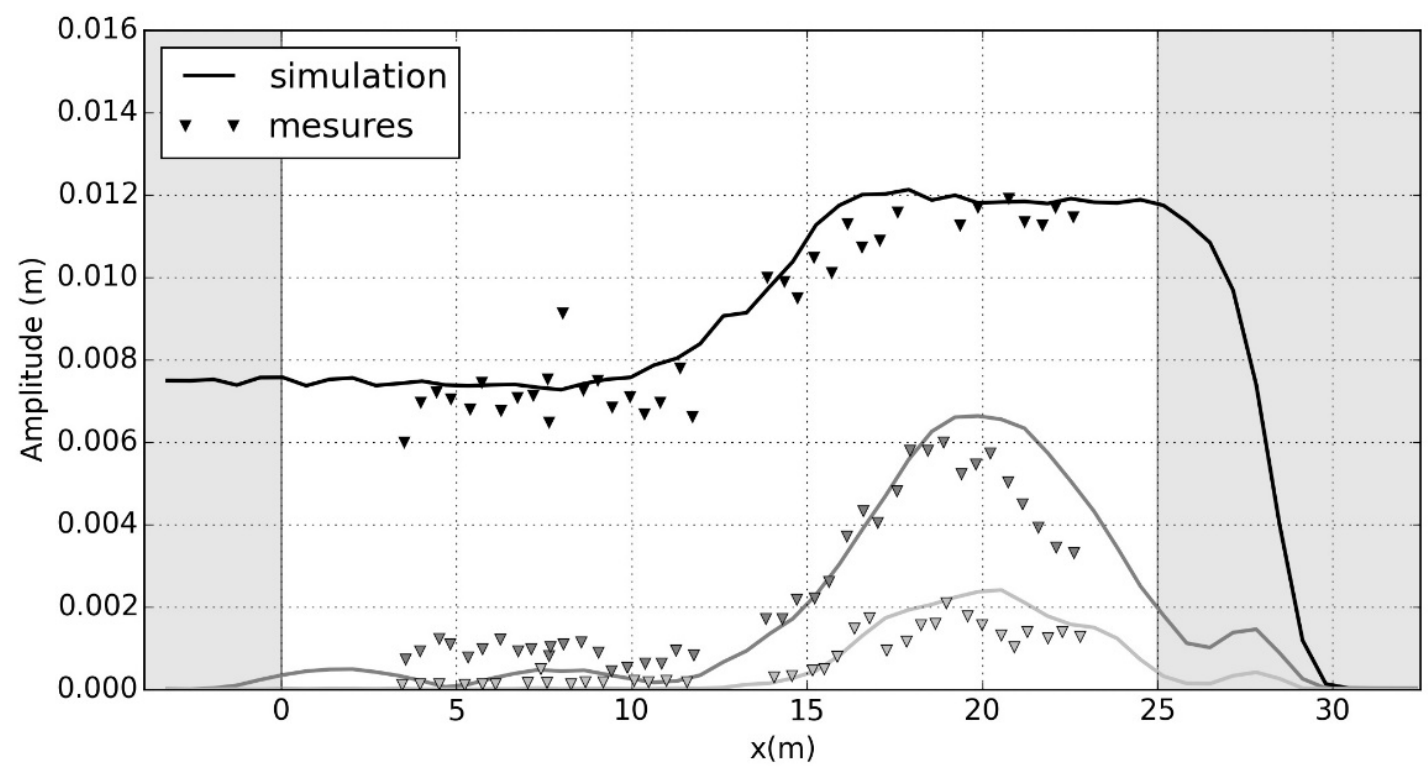

Figure 6. Evolution spatiale des amplitudes des trois premières harmoniques du premier cas test de WHALIN (1971), pour T=2 s et $a=0.0075 \mathrm{~m}$. Les zones grisées correspondent aux zones de relaxation utilisées dans le modèle, et les trois couleurs correspondent aux harmoniques 1 (noir), 2 (gris foncé) et 3 (gris clair).

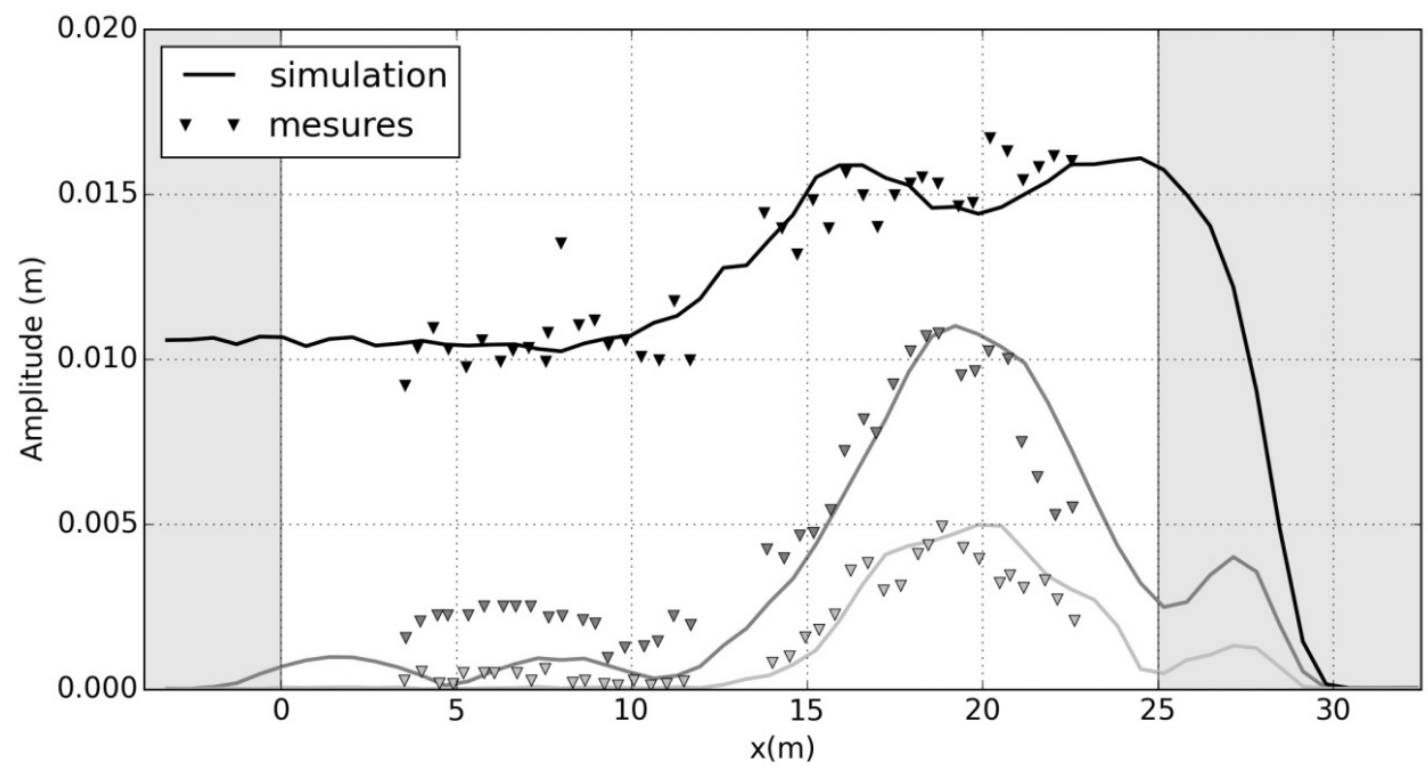

Figure 7. Evolution spatiale des amplitudes des trois premières harmoniques du second cas test de WHALIN (1971), pour $T=2$ s et a $=0.0106 \mathrm{~m}$. Les zones grisées correspondent aux zones de relaxation utilisées dans le modèle, et les trois couleurs correspondent aux harmoniques 1 (noir), 2 (gris foncé) et 3 (gris clair). 
De plus, le transfert d'énergie vers les harmoniques supérieures n'est plus exactement compensé par la réfraction, d'où la diminution de l'amplitude de la première harmonique autour de $x=20 \mathrm{~m}$.

Ce cas test a permis de montrer les capacités du modèle à simuler les effets de réfraction-shoaling non-linéaires sur une telle bathymétrie 3D avec des effets de convergence très marqués.

\section{Conclusions et perspectives}

Le modèle numérique proposé, fondé sur une approche potentielle non-linéaire (équations surfaciques d'Euler-Zakharov), permet de modéliser les effets dispersifs et non-linéaires dans la transformation des vagues en zone côtière, sur une large gamme de profondeurs d'eau relatives. Les deux cas $1 \mathrm{DH}$ présentés ont permis de vérifier ces capacités dispersives et non-linéaires, notamment les transferts d'énergie entre les différentes harmoniques (libres ou liées) lors de leur génération et leur propagation. Les longueurs de battement des harmoniques 2 et 3 des expériences de CHAPALAIN et al. (1992) sont très bien restituées par le modèle. Lors de la transformation d'un spectre de vagues irrégulières sur un profil côtier réaliste (BECQ-GIRARD et al., 1999), le modèle montre un bon accord dans la dynamique d'évolution des pics harmoniques du spectre jusqu'à 5 fois la fréquence de pic.

De plus, l'utilisation d'une méthode utilisant des fonctions de bases radiales (RBF) pour l'extension du modèle à deux dimensions dans le plan horizontal a montré de bonnes performances quant à la représentation des effets non-linéaires sur le cas de deux des expériences 3D de WHALIN (1971) avec deux amplitudes différentes, où une bathymétrie de forme semi-circulaire provoque une convergence de l'énergie des vagues vers l'axe du bassin par réfraction-shoaling, avec à nouveau des effets nonlinéaires importants. Un des avantages de cette méthode RBF est la possibilité de travailler sur des semis de points irréguliers, et donc d'avoir une résolution variable et adaptable en $(x, y)$ pour les applications réelles.

La détermination du paramètre de forme utilisé pour la fonction multiquadrique peut nécessiter une phase de tests préalables assez importante. Des fonctions radiales moins régulières mais ne dépendant pas de paramètre de forme (BARNETT, 2015) font l'objet de tests en cours sur la validation de la version $2 \mathrm{DH}$ du modèle. D'autre part, les travaux futurs concernent la représentation de certains effets du déferlement et du runup sur des talus inclinés. La version $2 \mathrm{DH}$ du modèle doit également être parallélisée en vue de son optimisation pour des applications d'ingénierie côtière et portuaire.

\section{Références bibliographiques}

BARNETT G.A. (2015) A robust RBF-FD formulation based on polyharmonic splines and polynomials. $\mathrm{PhD}$ Thesis, University of Colorado. 
Développement d'un modèle numérique non-linéaire et dispersif pour la propagation des vagues en zone côtière : n01.13

BECQ-GIRARD F., FORGET P., BENOIT M. (1999) Non-linear propagation of unidirectional wave fields over varying topography. Coastal Engineering, Vol. 38, pp 91-113. https://doi.org/10.1016/S0378-3839(99)00043-5

BENOIT M., CHAZEL F. (2013). Validation expérimentale d'un modèle double-couche pour des vagues côtières non-linéaires et fortement dispersives. Revue Paralia, Vol. 6, pp 7.1-7.16. https://doi.org/10.5150/revue-paralia.2013.007

BENOIT M., RAOULT C., YATES M.L. (2014) Fully nonlinear and dispersive modelling of surf zone waves: non-breaking tests. Coastal Engineering Proceedings, 1(34), waves.15, 15-20 July 2014, Seoul (Korea). https://doi.org/10.9753/icce.v34.waves.15

BERKHOFF J.C.W. (1972) Computation of combined refraction-diffraction. Proceeding of the 13th International Conference on Coastal Engineering, ASCE, Vancouver (Canada), pp 470-490.

CHAPALAIN G., COINTE R., TEMPERVILlE A. (1992) Observed and modeled resonantly interacting progressive water-waves. Coastal Engineering, Vol. 16, pp 267300. https://doi.org/10.1016/0378-3839(92)90045-V

CHAZEL F., LANNES D., MARCHE F. (2011). Numerical simulation of strongly nonlinear and dispersive waves using a Green-Naghdi model. Journal of Scientific Computing, Vol. 48(1-3), pp 105-116. https://doi.org/10.1007/s10915-010-9395-9

KIRBY J.T. (2003). Boussinesq models and applications to nearshore wave propagation, surf zone processes and wave-induced currents. Advances in Coastal Modeling, V.C. Lakhan (ed), Elsevier, pp 1-41.

MADSEN P.A., MURRAY R., SORENSEN O.R. (1991). A new form of the Boussinesq equations with improved linear dispersion characteristics. Coastal Engineering, Vol. 15, pp 371-388. https://doi.org/10.1016/0378-3839(91)90017-B

MADSEN P.A., BINGHAM H.B., LIU H. (2002). A new Boussinesq method for fully nonlinear waves from shallow to deep water. Journal of Fluid Mechanics, Vol. 462, pp 1-30. https://doi.org/10.1017/S0022112002008467

MADSEN P.A., FUHRMAN D.R., WANG B. (2006). A Boussinesq-type method for fully nonlinear waves interacting with a rapidly varying bathymetry. Coastal Engineering, Vol. 53, pp 487-504. https://doi.org/10.1016/j.coastaleng.2005.11.002

NWOGU O.G. (1993). Alternative form of Boussinesq equations for nearshore wave propagation. Journal of Waterway, Port, Coastal and Ocean Engineering, Vol. 119, pp 618-638. https://doi.org/10.1061/(ASCE)0733-950X(1993)119:6(618)

RAOULT C., BENOIT M., YATES M.L. (2014) Etude et qualification d'un modèle numérique complètement non-linéaire et dispersif pour les vagues en zone côtière. XIIIème Journées Nationales Génie Côtier Génie Civil, 2-4 juillet 2014, Dunkerque (France), pp 159-168. https://doi.org/10.5150/jngcgc.2014.018

RAOULT C., BENOIT M., YATES M.L. (2016) Validation of a fully nonlinear and dispersive wave model with laboratory non-breaking experiments. Coastal Engineering, Vol. 114, pp 194-207. https://doi.org/10.1016/j.coastaleng.2016.04.003 
n01.14 : Revue Paralia - Vol. 11 (2018)

SHAO Y-L., FALTINSEN O.M. (2014) A harmonic polynomial cell (HPC) method for $3 D$ Laplace equation with application in marine hydrodynamics. Journal of Computational Physics, Vol. 274, pp 312-332. https://doi.org/10.1016/j.jcp.2014.06.021

TIAN Y., SATO S. (2008). A numerical model on the interaction between nearshore nonlinear waves and strong currents. Coastal Engineering Journal, Vol. 50(4), pp 369395. https://doi.org/10.1142/S0578563408001879

WHALIN R.W. (1971) The limit of applicability of linear wave refraction theory in a convergence zone. Technical report, DTIC Documents.

WRIGHT G.B., FORNBERG B. (2006) Scattered node compact finite difference-type formulas generated from radial basis functions. Journal of Computational Physics, Vol. 212, pp 99-123. https://doi.org/10.1016/j.jcp.2005.05.030

YATES M.L., BENOIT M. (2015) Accuracy and efficiency of two numerical methods of solving the potential flow problem for highly nonlinear and dispersive water waves. International Journal for Numerical Methods in Fluids. Vol. 77(10), pp 616-640. https://doi.org/10.1002/fld.3992

ZAKHAROV V.E. (1968). Stability of periodic waves of finite amplitude on the surface of a deep fluid. Journal of Applied Mechanics and Technical Physics, Vol. 9(2), pp 190-194. https://doi.org/10.1007/BF00913182 